\title{
THERMALLY INDUCED INTERMIXING OF InGaAs/GaAs SINGLE QUANTUM WELLS
}

\author{
A. KozANecki \\ Institute of Physics, Polish Academy of Sciences \\ Al. Lotników 32/46, 02-668 Warszawa, Poland \\ W.P. Gillin and B.J. Sealy \\ Department of Electronic and Electrical Engineering, University of Surrey \\ Guildford, Surrey GU1 5XH, UK
}

In this work interdiffusion and strain relaxation in $\operatorname{In}_{0.2} \mathrm{Ga}_{0.8} \mathrm{As} / \mathrm{GaAs}$ single quantum wells subjected to rapid thermal annealing have been studied using photoluminescence and Rutherford backscattering of $1.5 \mathrm{MeV} \mathrm{He}^{+}$ ions. It has been found that the diffusion coefficient of In atoms in GaAs, evaluated from the photoluminescence spectra for the assumed Gaussian well shapes, agrees within $30 \%$ with that obtained using Rutherford backscattering. Channeling angular scans, through the $\langle 110\rangle$ axial direction of the heterostructures indicate that strain relaxation in the intermixed wells is exclusively due to compositional shallowing of the wells.

PACS numbers: $61.80 . \mathrm{Mk}, 78.65 . \mathrm{Fa}$

Single quantum wells (SQW) grown off GaAs substrates are of great interest for optoelectronic devices. However, devices manufactured in these materials are very susceptible to thermally induced interdiffusion of the active region. This results in emission wavelengths shifted from their nominal values. Intermixing also results in non-square composition profiles which have to be taken into account when modeling devices such as lasers.

Photoluminescence (PL) is one of the principal techniques which have been used to study the effects of intermixing (see i.e. $[1,2]$ ). The interdiffusion coefficient can be evaluated from the PL spectra by modeling the diffusion and calculating theoretically the expected shift in the PL energy of excitons bound in the well [3]. This technique, however, relies on the assumption that the diffusion obeys Fick's law and has a diffusion coefficient independent of concentration gradient. In this work, apart from luminescence, ion beam analysis techniques (RBS and channeling) have been utilized to obtain direct experimental information on the shape of intermixed SQWs and the strain in ultrathin $(10 \mathrm{~nm}) \operatorname{In}_{0.2} \mathrm{Ga}_{0.8} \mathrm{As} / \mathrm{GaAs}$ SQWs.

The samples were grown by molecular beam epitaxy (MBE) and consisted of a $10 \mathrm{~nm} \cdot \mathrm{In}_{0.2} \mathrm{Ga}_{0.8} \mathrm{As} / \mathrm{GaAs} \mathrm{SQWs}$ grown off a GaAs substrate with a $30 \mathrm{~nm}$ 
capping layer. Rapid thermal annealing (RTA) of the samples covered with a $50 \mathrm{~nm} \mathrm{Si} \mathrm{N}_{4}$ protective layers was conducted in a double strip graphite heater in a nitrogen atmosphere. For RBS/channeling measurements using a $1.5 \mathrm{MeV} \mathrm{He}{ }^{+}$ ion beam the samples were mounted on a three axis goniometer. The backscattered particles were detected at an angle of $150^{\circ}$.

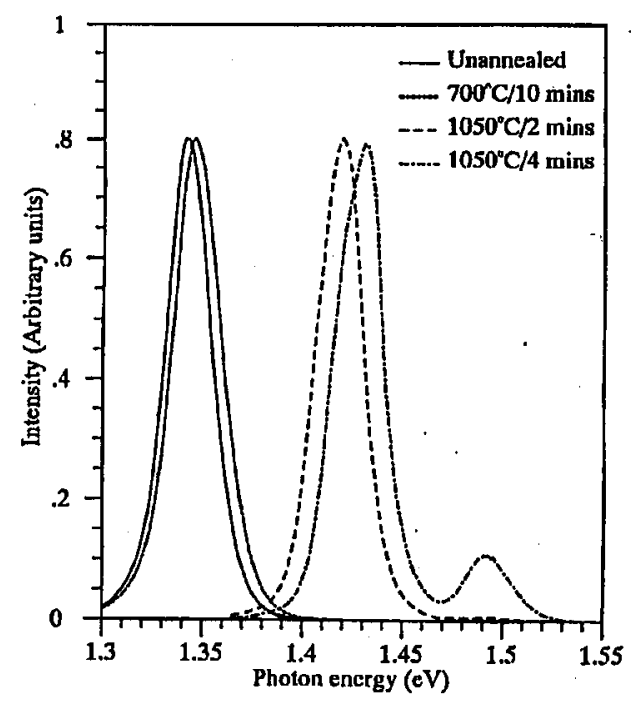

Fig. 1. Photoluminescence spectra of RTA annealed GaAs/In $0.2 \mathrm{Ga0.8}$ As/GaAs SQWs.

Figure 1 presents the PL spectra at $77 \mathrm{~K}$ of SQWs RTA annealed at selected temperatures. In agreement with the previous observations $[2,4]$ the PL peak shifts to higher energies, indicating the shallowing and broadening of the SQW. An emission band at $1.495 \mathrm{eV}$ which appeared in the PL spectrum after 4 minute annealing at $1050^{\circ} \mathrm{C}$ originates most probably in the GaAs substrate. It suggests that quantum confinement in SQW has been reduced. The diffusion length $L_{\mathrm{d}}$ values have been extracted from the PL spectra using a method of Gillin et al. $[3,5]$. The $L_{\mathrm{d}}$ values are $1.2,8.8$, and $10.5 \mathrm{~nm}$ for $700^{\circ} \mathrm{C}, 1050^{\circ} \mathrm{C}(120 \mathrm{~s})$ and $1050^{\circ} \mathrm{C}(4 \mathrm{~min})$, respectively.

In Fig. 2 the In profiles measured with Rutherford backscattering (RBS) for the same samples as in Fig. 1 have been presented. A statistical analysis of the In profiles shows that the shape of the intermixed well can be approached with a Gaussian function (skew $=0$ ) in agreement with the assumptions made in the analysis of the PL spectra. The diffusion lengths of 5 and $7 \mathrm{~nm}$ for $100 \mathrm{~s}$ and $4 \mathrm{~min}$ annealing at $1050^{\circ} \mathrm{C}$, respectively, have been calculated from the second moments of In distributions. The maximum In contents of $12 \%$ in the sample annealed at $1050^{\circ} \mathrm{C}$ (4 min) estimated from the RBS data in Fig. 2, agrees well with that $(\approx 13 \%)$ calculated from its PL spectrum (Fig. 1).

Figure 3 presents the angular scans through the $\langle 110\rangle$ axial channel in the (100) plane for as-grown and annealed SQW (the integrated In signal), and a ref- 


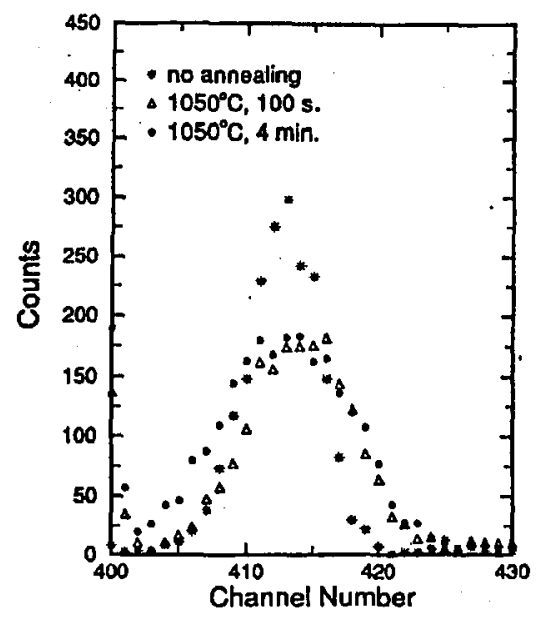

Fig. 2. Indium distributions in as-grown and annealed structures (same as in Fig. 1) as measured with RBS.

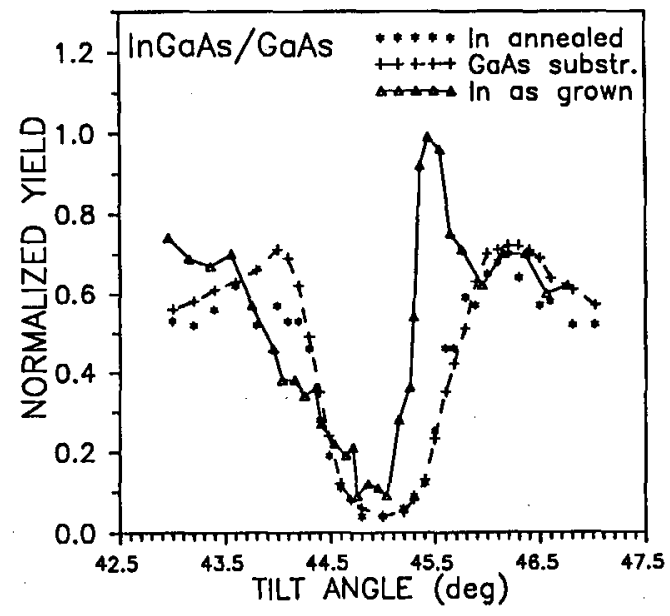

Fig. 3. Angular scans through the $\langle 110\rangle$ axial channel in the (100) plane for as-grown and annealed at $1050^{\circ} \mathrm{C}$ SQWs. Scan for GaAs substrate is given for reference.

erence signal from GaAs substrate. The In scan profile for an as-grown structure is asymmetric, but its absolute minimum position almost coincides with the minimum of the substrate angular scan. Asymmetry in the In angular scan is caused by beam steering in the top GaAs overlayer [6,7]. Beam steering effect makes quantitative analysis of the strain hardly feasible, as shift between the absolute minima of the substrate and SQW angular scans does not reflect the "kink angle" between the $\langle 110\rangle$ axes in SQW and the substrate, oppositely to the results of channeling scans of thin InGaAs [6, 8] and GaAsSb [9] epilayers deposited on GaAs. 
The $\langle 110\rangle$ angular scans for SQW annealed at $1050^{\circ} \mathrm{C}$ and $\mathrm{GaAs}$ substrate are almost overlapping (Fig. 3). It clearly indicates that the In atoms are located at substitutional positions. The generation of a high concentration of misfit dislocations can in essence be excluded, as the minimum backscattering yields of the annealed SQW and the substrate also coincide. This conclusion is confirmed by optical measurements (Fig. 1), which show that the structures have not been degraded by the annealing.

Summarizing: an agreement between the diffusion length measured using RBS and that evaluated from the PL spectra has been obtained. The experimental data have given evidence that strain relaxation in annealed SQWs is solely due to interdiffusion.

\section{References}

[1] W.D. Laidig, N. Holonyak, Jr., M.D. Camras, K. Hess, J.J. Colman, P.D. Dapkus, J. Bardeen, Appl. Phys. Lett. 38, 776 (1981).

[2] See papers in special issue of Opt. Quantum Electron. 23, No. 7 (1991).

[3] I.V. Bradley, W.P. Gillin, K.P. Homewood, R.P. Webb, J. Appl. Phys. 73, 1686 (1993).

[4] B. Elman, E.S. Koteles, P. Melman, C. Jagannath, C.A. Armiento, M. Rothman, J. Appl. Phys. 68, 1351 (1990).

[5] W.P. Gillin, I.V. Bradley, L.K. Howard, R. Gwilliam, K.P. Homewood, J. Appl. Phys. 73, 3782 (1993).

[6] R. Flagmayer, H. Krause, G. Oelgart, G. Rohde, Phys. Status Solidi A 124, 265 (1991).

[7] J.L.E. Stevens, B.J. Robinson, J.A. Davies, D.A. Thompson, T.E. Jackman, J. Appl. Phys. 65, 1510 (1989).

[8] R. Flagmayer, M. Horicke, Nucl. Instrum. Methods Phys. Res. B 30, 219 (1988).

[9] S.T. Picraux, L.R. Dawson, J.Y. Tsao, B.L. Doyle, S.R. Lee, Nucl. Instrum. Methods Phys. Res. B 33, 891 (1988). 